\title{
Implementing a pharmacovigilance program to evaluate cutaneous adverse drug reactions in an antiretroviral access program
}

\author{
Tinashe Mudzviti ${ }^{1}$, Marvelous Sibanda ${ }^{1}$, Samuel Gavi ${ }^{2}$, Charles Chiedza Maponga ${ }^{1,3}$, \\ Gene D. Morse ${ }^{3}$ \\ ${ }^{1}$ School of Pharmacy, University of Zimbabwe, Harare, Zimbabwe \\ ${ }^{2}$ Department of Clinical Pharmacology, College of Health Sciences, University of Zimbabwe, Harare, Zimbabwe \\ ${ }^{3}$ School of Pharmacy and Pharmaceutical Sciences and the Center of Excellence in Bioinformatics and Life \\ Sciences, SUNY, University at Buffalo, Buffalo, NY, United States of America
}

\begin{abstract}
Introduction: Cutaneous adverse drug reactions (cADRs) can cause significant morbidity and distress in patients, especially in the HIVinfected population on antiretroviral therapy. Adverse drug reaction monitoring and ascertaining causality in resource-limited settings remain serious challenges. This study was conducted to evaluate causality and measure the incidence of cADRs in HIV-infected patients on highly active antiretroviral therapy. The study was also designed to test a three-step approach in the monitoring and evaluation of ADRs in resourcelimited settings.

Methodology: A retrospective review of patient medical records was performed at the Parirenyatwa Family Care Centre, Harare, Zimbabwe. Cases of cADRs were reported to the Medicines Control Authority of Zimbabwe, the main drug regulating body in Zimbabwe, for assessment and causality classification.

Results: We reviewed 221 randomly selected patient records to determine whether any diagnoses of cADRs were made by clinicians. Causality assessment revealed that $13.1 \%$ of cADRs were due to an offending agent in the antiretroviral therapy versus an initial incidence of $17.6 \%$ which had been determined by the physicians.

Conclusions: cADRs had an incidence of $13.1 \%$ within the population under study due to non nucleoside reverse transcriptase inhibitors (NNRTIs). Most reactions were caused by the NNRTIs which contributed $72.4 \%$ of all cADRs. A panel of experts from the drug regulatory authority can be used as an implementation based mechanism in ascertaining causality objectively in settings where resources are constrained.
\end{abstract}

Key words: pharmacovigilance; antiretrovirals; cutaneous adverse drug reaction

J Infect Dev Ctries 2012; 6(11):806-808.

(Received 06 February 2011 - Accepted 17 January 2012)

Copyright (C) 2012 Mudzviti et al. This is an open-access article distributed under the Creative Commons Attribution License, which permits unrestricted use, distribution, and reproduction in any medium, provided the original work is properly cited.

\section{Introduction}

HIV infected patients have a higher risk of developing cutaneous reactions than the general population, which has a significant impact on patients' current and future treatment options. The severity of cutaneous adverse reactions (cADRs) varies greatly, and HIV-infected patients initiating antiretroviral treatment can frequently show a wide variety of adverse drug reactions [1]. Various patient- and drugrelated factors contribute to the risk of adverse drug reactions [2]. Drug hypersensitivity in HIV-1 infected patients has been found to be about 100 times more common than in the general populace [3].

Hypersensitivity typically manifests as erythematous maculopapular, pruritic and confluent rash with or without fever. Rash is most prominent on the body and arms and begins after one to two weeks of therapy. Constitutional features are often prominent and can precede rash (with abacavir) or occur without rash. According to Carr et al., Stevens Johnson syndrome or toxic epidermal necrolysis have been found to develop in less than $0.3 \%$ of patients on highly active antiretroviral therapy (HAART) [3].

According to Holly et al. [4], a patient must be initiated on antiretroviral (ARV) drugs with nonoverlapping toxicities and a small risk of interaction with other existing treatments. Post adverse reaction re-challenge should be medically supervised but is contraindicated with hypersensitivity to abacavir, mucosal involvement or grade 3-4 rashes [4]. The ultimate goal in a pharmacovigilance program is always to discontinue the offending medication if 
possible. Individuals with cutaneous drug eruptions are often very ill patients taking a large number of medications, many of which are essential for their survival. However, all nonessential medications should be discontinued. Knowing the common eruption-inducing medications may help in identifying the offending drug. The decision of whether to continue to administer a drug that is known or assumed to be the cause of a reaction will be influenced by the following four key factors: the severity and probable course of the reaction; the disease for which the drug was prescribed; ease or difficulty with which the reaction can be managed; and the availability of chemically unrelated drugs with similar pharmacologic properties [5].

Ascertaining the causality of suspected adverse drug reactions (ADRs) still remains a challenge in resource-limited settings, mostly due to the unavailability of qualified personnel in health institutions and the inconsistent laboratory support resulting in poor implementation of pharmacovigilance programs. This study was designed to test the effectiveness of utilizing available resources in a three-step approach to ascertain causality of ADRs. The study also aimed to measure the incidence of cADRs in HIV-infected patients during ART.

\section{Methodology}

The study was conducted as a retrospective record review in a population of HIV-infected adults on ART. The study was performed at the Family Care Centre (FCC), ART clinic in Harare, Zimbabwe. The FCC is integrated into the outpatient department for patients to receive antiretrovirals free of charge and for the treatment of opportunistic infections. Only data for patients who were above 18 years of age who had been initiated on ART in 2008 was collected for inclusion in the study. All data for patients who had been commenced on ART with a pre-existing skin condition was excluded. Patients with no follow-up record post initiation of ART were excluded from the study.

The three-step approach was a process which involved an initial documentation of patient data on patient charts by the physician. The next step involved extraction of data from the respective patient charts by the study pharmacist. Data extracted from the charts was then submitted to the Medicines Control Authority of Zimbabwe (MCAZ) for an independent causality assessment and results of this approach were compared with results obtained in literature.

From a population of HIV-infected adults, 221 medical records of patients who started on a new drug regimen were randomly selected. Cases of cutaneous ADRs were recorded on MCAZ adverse drug reaction forms which were subsequently sent to the MCAZ for assessment and causality classification [6]. The MCAZ is the sole drug regulatory body in Zimbabwe responsible for regulatory issues relating to medicines. The MCAZ has an ADR committee that meets once a month to discuss issues relating to pharmacovigilance in Zimbabwe.

Data recorded included age at time of reaction, weight, sex, date the reaction started, date the reaction ceased, description of the skin reaction, type of treatment given, outcome of treatment, severity of reaction, generic name of drug suspected to have caused the reaction, drug indication, route of administration and dosage form of drug, other drugs concomitantly used by patient, and laboratory test results (CD4 count just before starting on the drug new regimen). Written consent was given by all the study participants. The study was approved by the Joint Research Ethics Committee of Parirenyatwa Hospital and the University of Zimbabwe.

\section{Results}

A total of 221 (125 female and 96 male) patients' medical records were reviewed for those who started on a new ARV drug regimen between January 2008 and December 2008. The mean age of the patients was 40.6 years $(\mathrm{SD}=11.2$ years) (Table 1$)$.

Causality assessment by the MCAZ revealed 5 cases $(2.3 \%)$ to be probable, $24(10.9 \%)$ to be possible, $4(1.8 \%)$ to be unlikely, and 6 cases were unclassified due to the unavailability of patient's clinical data relating to the assumed ADR.

Cases classified as probable or possible by the MCAZ adverse drug reaction assessment committee (WHO causality classification) were selected for further analysis. Hence a total of 29 cases (13.1\%) were selected. Of the 29 cases $21(72.4 \%)$ were due to the non-nucleoside reverse transcriptase inhibitors (NNRTIs), nevirapine and efavirenz. Among the NNRTIs 3 cases (14.2\%) were presented as erythematous rash, 6 cases $(28.5 \%)$ presented as papular rash, 6 cases as generalized skin rash, and 2

Table 1. Patient demographics

\begin{tabular}{|l|l|}
\hline Female & $125(57 \%)$ \\
\hline Age (mean, SD) & $40.6(11.2)$ \\
\hline Physician-determined cADRs & $39(17.6 \%)$ \\
\hline
\end{tabular}


Table 2. Comparison of incidences of cADRs (Literature vs Physician's suspicions vs 3-step approach)

\begin{tabular}{|c|c|c|}
\hline $\begin{array}{c}\text { Literature } \\
\text { values }\end{array}$ & $\begin{array}{c}\text { 3-Step } \\
\text { approach }\end{array}$ & Physician determined \\
\hline $13.3 \%[7]$ & $13.1 \%$ & $17.6 \%$ \\
\hline
\end{tabular}

cases $(9.5 \%)$ presented as Stevens-Johnson syndrome, of which both were due to nevirapine. The remaining 8 cases $(27.6 \%)$ were due to other drugs, with one hypersensitivity (urticarial) reaction due to abacavir and 7 generalized skin rashes.

\section{Discussion}

The incidence of cADRs was $13.1 \%$ as determined by the ADR committee. This result was obtained by considering the probable and the possible result that was obtained after deliberation. The results obtained by the three-step approach are comparable to those reported in literature (13.3\%) [7]. The three-step approach is more suited to the resource-constrained setting where there are limited medical personnel. In peri-urban and rural settings where there might be no qualified health personnel, an individual need only record all the data routinely put in the patient charts. In the second step, a clinically minded health professional (in this instance a pharmacist) collects the data from the various treatment centres and then in the third step submit this data to a central body for evaluation. This type of an approach will generate reliable data while utilizing the available structures and the limited human resources.

The results of this study also revealed the need to identify specific populations at risk of cADRs and predisposing factors in different populations. The incidence rates for cADRs due to nevirapine also warrant the need for developing a biomarker suitable for identifying individuals who are at a higher risk of reacting to the drug. With increasing access to ART in developing countries and the increase in the use of nevirapine, such an intervention could prove to be a useful tool in decreasing morbidity and mortality due to ADRs.

\section{Acknowledgements}

The authors are grateful to the International Center for HIV/AIDS Pharmacotherapy Research and Training (ICHAPRT) for technical assistance. We are particularly grateful to the PARI support group for the excellent collaboration. Tinashe Mudzviti was partially supported by grant \# 5U2RTW007367-05 from the Fogarty International Center, National Institutes of Health (NIH-USA), and through the International Clinical, Operational and Health Services and Training Award (ICOHRTA) Programme, BIMR (award \# U2RTW007367).

This manuscript was supported by Award Number D43TW007991 from the Fogarty International Center. The content is solely the responsibility of the authors and does not necessarily represent the official views of the Fogarty International Center or the National Institutes of Health.

\section{References}

1. Centers for Disease Control and Prevention (2009) Bacterial coinfections in lung tissue specimens from fatal cases of 2009 pandemic influenza A (H1N1) - United States, May-August 2009. MMWR Morb Mortal Wkly Rep 58: 1071-1074.

2. Centers for Disease Control and Prevention (2009) Intensivecare patients with severe novel influenza A (H1N1) virus infection - Michigan, June 2009. MMWR Morb Mortal Wkly Rep 58: 749-752.

3. Centers for Disease Control and Prevention (2009) Hospitalized patients with novel influenza A (H1N1) virus infection - California, April-May, 2009. MMWR Morb Mortal Wkly Rep 58: 536-541.

4. Mauad T, Hajjar LA, Callegari GD, da Silva LF, Schout D, Galas FR, Alves VA, Malheiros DM, Auler JO Jr, Ferreira AF, Borsato MR, Bezerra SM, Gutierrez PS, Caldini ET, Pasqualucci CA, Dolhnikoff M, Saldiva PH (2010) Lung Pathology in Fatal Novel Human Influenza A (H1N1) Infection. Am J Respir Crit Care Med. 181: 72-79.

\section{Corresponding author}

Tinashe Mudzviti

School of Pharmacy

University of Zimbabwe

PO Box MP167

Mount Pleasant, Harare, Zimbabwe

Telephone: +263772290511; Fax: +2634788272

Email: tmudzviti@yahoo.co.uk

Conflict of interests: No conflict of interests is declared. 